\title{
Diltiazem alone and combined with nitroglycerin: effect on normal and diseased human coronary arteries
}

\author{
O. M. Hess, H. Nonogi, A. Bortone, J. E. Gage, J. Grimm and H. P. Krayenbuehl \\ Department of Internal Medicine, Medical Policlinic, Cardiology, University Hospital, Zurich, \\ Switzerland
}

KEY WORDS: Coronary vasomotion, quantitative coronary arteriography, coronary artery disease, intracoronary diltiazem, nitroglycerin.

The vasodilatory effect of diltiazem and nitroglycerin on the large epicardial coronary arteries was evaluated in 26 patients with coronary artery disease. The luminal area of a normal and a stenotic coronary artery was determined at rest, after intracoronary administration of diltiazem, during submaximal exercise as well as $5 \mathrm{~min}$ after $1.6 \mathrm{mg}$ sublingual nitroglycerin using biplane quantitative coronary arteriography. Twelve patients with no pretreatment prior to the exercise test served as group 1 (controls) and 14 patients with intracoronary administration of 2 to $3 \mathrm{mg}$ diltiazem prior to the exercise test as group 2.

Normal vessel: In the control group luminal area increased significantly during exercise (+23\%, $P<0.01)$ and after sublingual administration of nitroglycerin ( $+40 \%, P<0.001)$. In group 2 luminal area increased after intracoronary administration of diltiazem $(+19 \%, P<0.01)$, during bicycle exercise $(+23 \%, P<0 \cdot 001)$ and after sublingual administration of nitroglycerin $(+39 \%, P<0 \cdot 001)$.

Stenotic vessel: In the control group luminal area decreased significantly $(-29 \%, P<0.001)$ during bicycle exercise but increased after sublingual administration of nitroglycerin at the end of the exercise test $(+12 \%$, NS vs. rest). In group 2 intracoronary administration of diltiazem was associated with a mild increase in stenosis area $(+11 \%, P<0.05)$. There was a further increase in stenosis area during bicycle exercise $(+23 \%, P<0.001$ vs. rest) and after sublingual nitroglycerin $(+32 \%, P<0.001)$. Coronary vasodilation of the stenotic segment was, however, significantly more pronounced after sublingual nitroglycerin in group 2 than 1 ( $+32 \%$ versus $12 \%, P<0.05)$.

Thus, it is concluded that diltiazem prevents exercise-induced coronary vasoconstriction of the stenotic vessel segment probably due to its direct vasorelaxing action on the smooth vasculature. Diltiazem combined with nitroglycerin elicits an additive effect on coronary vasodilation of the stenotic vessel segments but not on the normal coronary arteries. The exact mechanism of this additive effect is not clear but might be due to the combined action of the two vasoactive drugs with different mode of action.

\section{Introduction}

Previous studies have shown that normal coronary arteries constrict during isometric exercise but dilate during dynamic exercise ${ }^{|1-3|}$. The factors which regulate coronary vasomotion during isometric and dynamic exercise are not yet fully understood but certainly involve

Address for correspondence: Otto M. Hess, M.D., Medical Policlinic, Cardiology, University Hospital, Raemistrasse 100, CH-8091 Zurich, Switzerland. haemodynamic variables such as heart rate, perfusion pressure and coronary vascular resistance, metabolic demands, neurohumoral factors and endothelium-derived vasoactive substances. Coronary vasomotor tone is the result of a complex interplay of all these factors which are modified in the presence of coronary obstructions with changes in myocardial blood flow. The purpose of the present study was to evaluate the effect of intracoronary administration of diltiazem on coronary vasomotion of normal and stenotic coronary arteries at rest and 
during dynamic exercise in patients with coronary artery disease.

\section{Patients and methods}

Twenty-six patients with a mean age of 55 years (range from 36 to 67 years) were included in the present study. A history of angina pectoris and/or ST-segment depression $\geqslant 0.1 \mathrm{mV}$ in the upright bicycle exercise test was present in all patients. The patients were recruited on a consecutive basis when a history of stable angina pectoris with no signs of coronary vasospasm and a clearly visible coronary artery stenosis for quantitative evaluation was present. The first 12 patients constituted the control group and the following 14 patients the diltiazem group. There were no significant differences between the two groups in regard to clinical findings or baseline haemodynamics. All medication was stopped at least 12 to $24 \mathrm{~h}$ before cardiac catheterization.

\section{STUDY PROTOCOL}

After the diagnostic coronary arteriography (Judkins technique) an interval of at least $10 \mathrm{~min}$ was allowed for dissipation of the effects of the contrast medium on coronary vasomotion. After the patient's feet were attached to the bicycle ergometer biplane, coronary arteriography was performed ${ }^{[3.4]}$. Aortic and pulmonary artery pressure were recorded immediately before coronary arteriography. Biplane coronary arteriography was repeated at the end of each level of exercise which was begun at 50 to $75 \mathrm{~W}$ and was increased every $2 \mathrm{~min}$ in increments of 25 to $50 \mathrm{~W}$. The exercise was terminated because of anginal pain, fatigue or ST-segment depression of more than $0.2 \mathrm{mV}$. Two to $3 \mathrm{mg}$ diltiazem were infused prior to the exercise test in group 2 (= diltiazem group) into the coronary artery which was selected for quantitative analysis. At the end of the exercise test $1.6 \mathrm{mg}$ sublingual nitroglycerin was administered in both groups and biplane coronary arteriography was repeated $5 \mathrm{~min}$ thereafter. There were no complications related to the procedure in any of the 26 patients.

\section{QUANTITATIVE CORONARY ARTERIOGRAPHY}

The currently used technique for quantitation of coronary artery stenoses has been described elsewher $^{[3,4]}$. The evaluation was performed in a blinded fashion. A normal and a stenotic vessel segment were traced manually 4 to 6 times and the results were averaged to reduce sampling error. The tracings were digitized and analysed on a PDP 11/34 computer. Interobserver variability was $9.3 \%$ of the mean vessel area for monoplane and $7.9 \%$ for biplane measurements ${ }^{[3,4]}$.

\section{STATISTICS}

Comparisons of pressure and angiographic data in response to diltiazem, bicycle exercise and sublingual nitroglycerin was performed by a two-way analysis of variance for repeated measurements. Comparisons between group 1 and 2 were carried out by an unpaired Student $t$-test.

\section{Results}

\section{PRESSURE MEASUREMENTS}

Mean aortic and mean pulmonary artery pressure (Fig. 1) increased significantly during bicycle exercise from $87 \mathrm{mmHg}$ to $107 \mathrm{mmHg}$ $(P<0.001)$ and from 26 to $47 \mathrm{mmHg}$, respectively in the control group. In group 2 mean aortic pressure decreased significantly from $100 \mathrm{mmHg}$ to $89 \mathrm{mmHg}(P<0.01)$ after intracoronary administration of diltiazem and increased slightly to $101 \mathrm{mmHg}$ (NS vs. rest) during exercise. Mean pulmonary artery pressure remained unchanged after intracoronary administration of diltiazem but increased significantly from $22 \mathrm{mmHg}$ to $37 \mathrm{~mm} \mathrm{Hg}(P<$ $0 \cdot 001$ ) during bicycle exercise. Sublingual administration of $1.6 \mathrm{mg}$ nitroglycerin was associated with a fall in mean aortic pressure in both groups but the decrease in mean aortic pressure was statistically significant $(P<0.001$ vs. rest) only in group 2 . Mean pulmonary artery pressure dropped in both groups after sublingual nitroglycerin but significantly $(P<0.01)$ more in group $2(15 \mathrm{mmHg})$ than $1(24 \mathrm{mmHg})$.

\section{ANGIOGRAPHIC MEASUREMENTS}

The individual changes in coronary luminal area of the stenotic vessel segment are shown in Fig. 2 and the mean changes \pm 1 standard error for both normal and stenotic vessel segments are depicted in Fig. 3. The individual stenoses (Fig. 2) showed a tendency to increase its area after intracoronary administration of diltiazem and during subsequent bicycle exercise. There was a further increase in most patients ( 8 out of 


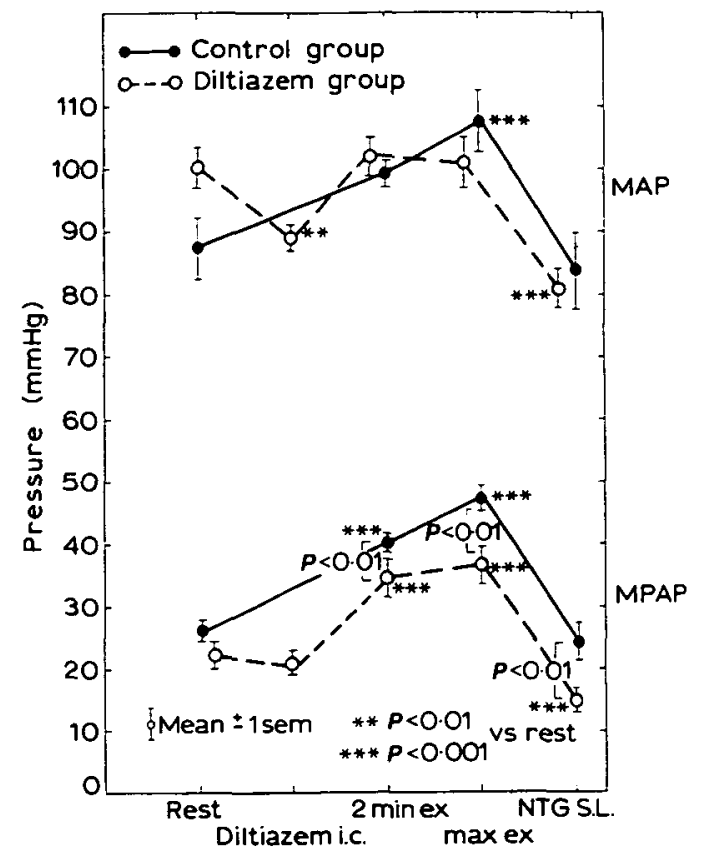

Figure 1 Mean aortic pressure (MAP) and mean pulmonary artery pressure (MPAP) in 26 patients with coronary artery disease and exercise-induced angina pectoris. Twelve patients with no pretreatment prior to exercise test served as control group (dots with solid lines) and 14 patients with intracoronary administration of 2-3 mg diltiazem prior to exercise as group 2 (circles with dashed lines). Both mean aortic and mean pulmonary artery pressure rose significantly during exercise (ex) in the control group but aortic pressure dropped after intracoronary administration of diltiazem and did not rise compared to rest during exercise in group 2. Mean pulmonary artery pressure rose significantly in group 2 during exercise but was significantly lower than in group $1.2 \mathrm{~min}$. ex, $2 \mathrm{~min}$. of exercise; max. ex, maximal exercise; NTG s.l., sublingual nitroglycerin.

14) after sublingual administration of nitroglycerin.

Normal coronary vessel segments (Fig. 3) showed coronary vasodilation during exercise $(+23 \%, P<0.001)$ and after sublingual administration of nitroglycerin $(+40 \%, P<0.001)$ in the control group. In group 2 there was coronary vasodilation $(+19 \%, P<0.01)$ after intracoronary administration of diltiazem and during bicycle exercise $(+23 \%, P<0.001)$. Sublingual nitroglycerin further dilated the normal vessel segments to a similar extent as in the control group $(+39 \%, P<0.001)$.

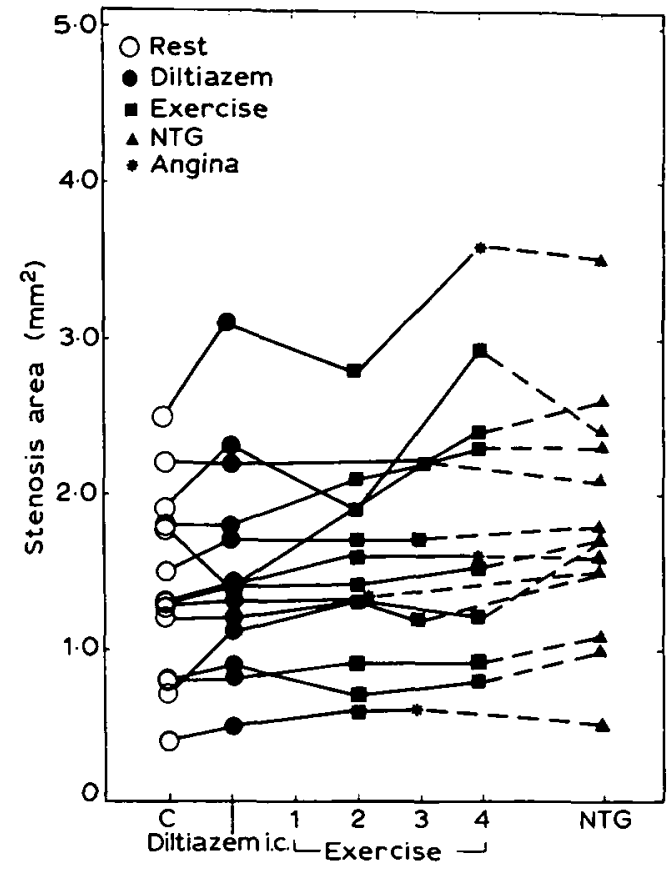

Figure 2 Changes in stenosis area for all 14 patients with intracoronary administration of $2-3 \mathrm{mg}$ diltiazem (group 2) prior to the exercise test. Given are the data at rest (C), after intracoronary (i.c.) administration of diltiazem, at each minute of the exercise test as well as 5 min after $1.6 \mathrm{mg}$ sublingual nitroglycerin. The occurrence of angina pectoris during exercise is indicated by an asterisk. There is a tendency to increase coronary stenosis area after intracoronary diltiazem, during bicycle exercise and after sublingual nitroglycerin.

Stenotic coronary vessel segments (Fig. 3) showed a completely different behaviour in groups 1 and 2 . In the control group there was exercise-induced coronary vasoconstriction $(-29 \%, P<0.001)$, whereas in group 2 intracoronary administration of diltiazem was associated with a mild increase $(+11 \%, P<0.05)$ and bicycle exercise with a significant increase in stenosis area $(+23 \%, \quad P<0.001$ vs. rest). Sublingual administration of nitroglycerin led in both groups to coronary vasodilation (group 1: $+12 \%$, NS vs. rest; group $2:+32 \%, P<0.001$ vs. rest) which was, however, significantly more pronounced in group 2 than $1(P<0.05)$.

\section{Discussion}

Diltiazem represents a potent coronary vasodilator which reduces the influx of calcium via the 


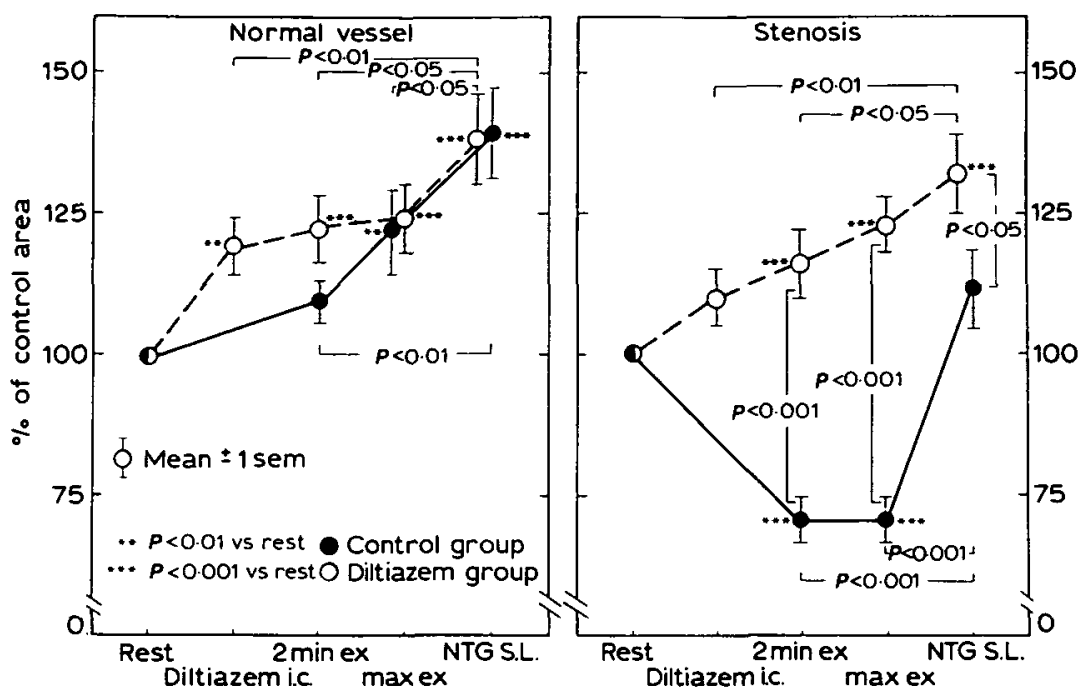

Figure 3 Changes in mean coronary luminal area of the normal (left) and stenotic (right) vessel segment. The data are given in percent of the control value. Twelve patients with no pretreatment prior to the exercise test served as controls (group 1; dots with solid lines) and 14 patients with intracoronary administration of $2-3 \mathrm{mg}$ diltiazem as group 2 (circles with dashed lines). The normal vessels showed coronary vasodilation during exercise in both groups, whereas the stenotic vessel demonstrated coronary vasoconstriction during exercise in the control group which was, however, prevented by intracoronary diltiazem in group 2 . Sublingual nitroglycerin elicited an additive effect on vasodilation of the stenotic $(+39 \%$ vs. $+12 \%, P<0 \cdot 05)$ but not of the normal vessel $(+39 \%$ vs. $+40 \%$, NS). Abbreviations see Fig. 1.

slow-calcium channels into the vascular smooth musculature. Nitroglycerin is also a potent coronary vasodilator which stimulates the guanylate cyclase with a consecutive rise in cyclic guanosine monophosphate in the smooth musculature which induces coronary vasodilation. Both drugs are well established in the treatment of patients with coronary artery disease and both have been shown to be highly efficient in the reduction of myocardial ischemia $^{[5,6]}$. Calcium antagonists and nitrates are often combined in the daily routine for an optimal anti-ischaemic effect in patients with angina pectoris although clinical trials did not show a better result for the combined regimen than for either drug alone ${ }^{[6]}$. Thus, the purpose of the present study was to evaluate the effect of diltiazem alone and combined with sublingual nitroglycerin on coronary vasomotion of normal and stenotic coronary arteries at rest and during dynamic exercise in patients with exerciseinduced ischaemia.

The effect of intracoronary administration of diltiazem on coronary vasomotion was similar for the normal and the stenotic vessel segment although the increase in luminal area was somewhat larger for the normal $(+19 \%$, $P<0.01)$ than for the stenotic vessel segment $(+11 \%$, NS). Exercise-induced coronary vasoconstriction was, however, prevented by intracoronary administration of diltiazem. The exact mechanism of exercise-induced stenosis vasoconstriction and its prevention by intracoronary diltiazem is not clear but might be due to the following 3 factors:

(1) Insufficient production (atherosclerotic plaques) of the endothelium-derived relaxing factor during exercise; (2) platelet aggregation with release of thromboxane $\mathrm{A} 2$ and serotonin due to turbulent blood flow; and (3) passive collapse of the free vessel wall within the stenosis due to high blood flow velocities during exercise (Venturi mechanism). Diltiazem could potentially restore endothelial function with a higher production of the endothelium-derived relaxing factor during exercise or could reduce 
turbulent blood flow and therefore platelet aggregation by coronary vasodilation and could prevent the passive collapse of the free vessel wall by a reduction in blood flow velocity (coronary vasodilation) during exercise. Which of these 3 factors is responsible for the exercise-induced vasoconstriction and which of the postulated effects of diltiazem prevents the exercise-induced vasoconstriction cannot be derived from the present study.

Sublingual administration of nitroglycerin at the end of the exercise test was associated with a significant increase in coronary luminal area of the normal vessel segments in both groups $(+40 \%$ in group 1 vs. $+39 \%$ in group $2, N S)$. The stenotic vessel segments showed, however, only a mild increase $(+12 \%$, NS vs. rest) in stenosis area after sublingual nitroglycerin in the control group, whereas stenosis area significantly increased $(+32 \%, P<0.001$ vs. rest) in patients pretreated with diltiazem (group 2). Apparently, the combination of both drugs seems to have an additive effect on coronary vasodilation of the stenotic but not of the normal vessel segment. The exact mechanism of this additive effect is not clear but might be related to the different mode of action of the two drugs as mentioned earlier: diltiazem reduces the calcium influx into the smooth muscle cell, whereas nitroglycerin increases the cyclic guanosine monophosphate in the vascular musculature. This combined action on the vascular smooth musculature of the diseased coronary artery appears to be responsible for maximal dilation of the stenotic vessel segments which exceeds that achieved by diltiazem or nitroglycerin ${ }^{[3]}$ alone.

\section{References}

[1] Brown BG, Bolson EL, Dodge HT. Dynamic mechanisms in human coronary stenosis. Circulation 1984; 70: 917-22.

[2] Brown BG, Lee AB, Bolson EL, Dodge HT. Reflex constriction of significant coronary stenosis as a mechanism contributing to ischemic left ventricular dysfunction during isometric exercise. Circulation $1984 ; 70: 18-24$.

[3] Gage JE, Hess OM, Murakami T, Ritter M, Grimm J, Krayenbuehl HP. Vasoconstriction of stenotic coronary arteries during dynamic exercise in patients with classic angina pectoris: reversibility by nitroglycerin. Circulation 1986; 73: 865-76.

[4] Gaglione A, Hess OM, Corin WJ, Ritter M, Grimm $J$, Krayenbuehl HP. Is there coronary vasoconstriction after intracoronary beta-adrenergic blockade in patients with coronary artery disease? J Am Coll Cardiol 1987; 10: 299-310.

[5] Boden WE, Bough EW, Reichmann MJ, Rich VB, Young PM, Korr KS, Shulman RS. Beneficial effects of high-dose diltiazem in patients with persistent effort angina on beta-blockers and nitrates: a randomized, double-blind placebo-controlled crossover study. Circulation 1985; 71: 1197-205.

[6] Hossack KF, Eldridge JE, Buckner K. Comparison of acute hemodynamic effects of nitroglycerin versus diltiazem and combined acute effects of both drugs in angina pectoris. Am J Cardiol 1986; 58: 722-6. 